Presentation

Published June 2, 2017

\title{
HIV Pathogenesis: Abstracts from the March 2017 Cleveland Immunopathogenesis Consortium Meeting
}

\section{Corresponding Author}

Michael M. Lederman

MXL6@case.edu
DOI

10.20411/pai.v2i2.210

The Cleveland Immunopathogenesis Consortium (CLIC) was launched in March 2004 by a small group of investigators (Ron Bosch, Jason Brenchley, Steven Deeks, Danny Douek, Zvi Grossman, Robert Kalayjian, Clifford Harding, Michael Lederman, Leonid Margolis, Miguel Quinones, Benigno Rodriguez, Rafick Sekaly, Scott Sieg, and Guido Silvestri) who were increasingly persuaded that immune activation was an important driver of HIV pathogenesis. We met around a chalk board and scribbled our models of pathogenesis, designed some experiments, then went back home to do them. We met again soon to review our new and unpublished findings that refined and shaped these models. The data presentations were short, informal and heavy on discussion. The model worked well, the consortium was productive and the meetings catalyzed numerous collaborations and scores of high impact papers. The CLIC (less formally, the Bad Boys of Cleveland [1]) has been meeting regularly since then. Consortium membership has expanded to include other investigators (some are listed in the presentations below). Whether the goal is to prevent the morbid complications of HIV infection, to understand the determinants of HIV persistence or the factors that protect from acquisition of infection, a more clear understanding of HIV immunopathogenesis is central. Here in this issue of Pathogens and Immunity is a brief summary of the most recent CLIC//BBC meeting held in Cleveland in March 2017. 


\section{PARTIAL NORMALIZATION OF MARKERS OF IMMUNE ACTIVATION AND TRANSIENT ELASTOGRAPHY DURING IFN FREE HCV THERAPY}

\section{Authors}

Lenche Kostadinova*, Carey L. Shive*, Elizabeth Zebrowski, Chelsey J. Judge, Kelsey Rife, Amy Hirsch, Anita Compan, Yngve Falck-Ytter, Daniel L Popkin, Donald D. Anthony

${ }^{*}$ contributed equally

\section{Lead Author}

Donald D. Anthony

\section{Lead Author's Institution}

Cleveland VA Medical Center, Cleveland, Ohio; Case Western Reserve University, Cleveland, Ohio

\section{Summary}

Plasma markers of immune activation during chronic HCV infection, including sCD14, sCD163, Mac2BP, IL-6, IP-10, and Autotaxin were measured before and over the course of Interferon free therapy for HCV, and results were compared to HCV level, serum markers of liver inflammation and function, and liver stiffness as measured by transient elastography. Results indicate select relationships between parameters before and those that change during HCV therapy, as well as those that reflect upon the HCV reservoir prior to therapy.

\section{LYMPH NODE RESIDENT PDCS ARE THE PRIMARY CELLULAR PRODUCER OF TYPE I IFN IN SIV INFECTION}

\section{Authors}

Reem Dawoud, Christine Grech, Amit Upadhyay, Amber Wolabaugh Nirav Patel, Greg Tharp, Simon Barratt-Boyes, Steven Bosinger

\section{Lead Author}

Steven Bosinger

\section{Lead Author's Institution}

Emory University, Atlanta, Georgia

\section{Summary}

In this study we purified plasmacytoid dendritic cells from SIV-infected rhesus macaques and sooty mangabeys, which remain resistant to AIDS. We used RNA-Seq to characterize the transcriptome of pDCs from blood and lymph nodes of both species. In concordance with prior observations, we observed that pDCs in SIV-infected Sooty Mangabeys had low to absent expression of Interferon Stimulated Genes, whereas ISGs were highly expressed in rhesus macaques. We found no detectable expression of IFNA, IFNB, or IFNW transcripts in pDCs purified from the blood of either species, however, we detected high levels of IFNA,B and W in pDCs purified from the Lymph Nodes of SIV-infected macaques. In contrast, IFNA/B/W could not be detected in $\mathrm{CD} 4+\mathrm{T}$ cells of SIV-infected macaques. These data indicate that the major driver of IFN responses in chronic HIV infection are lymph node resident pDCs. 


\title{
INDUCTION OF GI TRACT MICROBIAL DYSBIOSIS DOES NOT ACCELERATE SIV DISEASE PROGRESSION
}

\author{
Authors \\ Alex Ortiz, Carly Starke, Jacob Flynn, Carol Vinton, JC Mudd, Jason Brenchley \\ Lead Author \\ Jason Brenchley \\ Lead Author's Institution \\ Barrier Immunity Section, LPD, NIAID, NIH, Bethesda, Maryland
}

\section{Summary}

While GI tract microbial dysbiosis is observed in HIV-infected individuals, such dysbosis does not occur after experimental SIV infection of Asian macaques. Recent studies suggest that the dysbiosis observed in HIV-infection is, largely, attributed to lifestyle and not HIV infection itself. To assess if microbial dysbiosis accelerates SIV disease we experimentally induced bacterial GI tract dysbiosis in Asian macaques before and throughout SIV infection. The dysbiosis which we induced had no apparent effect on any virological or immunological attribute of SIV disease and the animals progressed to AIDS at rates similar to controls. Thus bacterial dysbiosis likely has little contribution to untreated HIV disease progression. Potential roles for dybiosis in immunopathogenesis of ARV-treated, HIV-infected individuals or in influencing susceptibility to infection are unclear.

\section{NON-INVASIVE MRI IMAGING OF LYMPHOID TISSUE DYSFUNCTION DURING SIV INFECTION}

\section{Authors}

Claire Deleage, Baris Turkbey, Marcelino L. Bernardo, Gregory Del Prete, Brandon Keele, Jeffrey Lifson, Jacob D. Estes

\section{Lead Author \\ Jacob D. Estes}

\section{Lead Author's Institution}

AIDS and Cancer Virus Program, Frederick National Laboratory, Leidos Biomedical Research Inc., Frederick, Maryland

\section{Summary}

Initiation of effective immune responses depends on the function of organized secondary lymphoid tissues, in particular lymph nodes. One of the important biological functions of lymph nodes is the filtration of the lymph and the capture of particulate antigens. Since HIV/SIV infection drives a significant and progressive lymphoid tissue fibrotic damage we sought to evaluate the ability of lymph nodes to capture particulate antigens over time during SIV infection using a novel imaging magnetic resonance imaging (MRI) approach. We found a rapid and progressive dysfunction of lymph nodes, with impaired uptake of particulate antigen into lymph nodes 
observed by MRI during SIV infection that was not restored after the 46 weeks of cART and 28 weeks of the anti-fibrotic pirfenidone. Lymph node impairment may limit the infected host's ability to mount effective immune responses and may limit drug penetration into the very tissue sites where the virus persists.

\section{NON-CANONICAL ACTIVATION OF CD8 T CELLS IN HIV INFECTION}

\section{Authors}

Stephen R. Morris, Soumya Panigrahi, Scott F. Sieg, Nicholas T. Funderburg, Souheil-Antoine Younes, Michael M. Lederman, and Michael L. Freeman

\section{Lead Author}

Michael L. Freeman

\section{Lead Author's Institution}

Case Western Reserve University, Cleveland, Ohio

\section{Summary}

We investigated the role of non-canonical CD2-mediated stimulation in vitro on CX3CR1+CD8 T cells from HIV-infected individuals. CD2-mediated stimulation resulted in elevated effector functions similar to levels induced by canonical TCR signaling. As a co-stimulator of TCR activation, CD2-mediated signals resulted in more robust responses than CD28-mediated co-stimulation.

\section{HIV INFECTION AND ITS TREATMENT ALTER LIPID SPECIES AND INFLAMMATORY MARKERS}

\section{Authors}

Martha Belury, Emily Bowman, Janelle Gabriel, Brandon Snyder, Manjusha Kulkarni, Marilly Palettas, Xiaokui Mo, Jordan E. Lake, Brian Clagett, Martin P. Playford, Adriana Andrade, Daniel R. Kuritzkes, Nehal N. Mehta, Michael M. Lederman, Nicholas T. Funderburg

\section{Lead Author}

Nicholas T. Funderburg

\section{Lead Author's Institution}

Ohio State University, Columbus, Ohio

\section{Summary}

The composition of the lipidome is altered in HIV-infection and changes with ART. Alterations in Saturated Fatty Acids were generally associated with inflammatory markers and may contribute to immune activation and comorbid disease pathogenesis. 


\title{
EFFECT OF CMV AND EBV REPLICATION ON INTESTINAL MUCOSAL GENE EXPRESSION AND MICROBIOME COMPOSITION OF HIV-INFECTED AND UNINFECTED INDIVIDUALS
}

\author{
Authors \\ Sara Gianella, Antoine Chaillon, Ece A. Mutlu, Phillip A. Engen, Robin M. Voigt, Ali Keshavar- \\ zian, John Losurdo, Prachi Chakradeo, Steven M. Lada, Masato Nakazawa, Alan L. Landay \\ Lead Author \\ Sara Gianella \\ Lead Author's Institution \\ University of California San Diego, San Diego, California

\section{Summary} \\ HIV-infection is associated with dramatic changes in the intestinal mucosa but the impact of oth- \\ er viral pathogens is unclear. Here we investigated the effect of intestinal CMV and EBV replica- \\ tion on the gut microbiome and mucosal cytokine expression of HIV-infected and -uninfected in- \\ dividuals. CMV and EBV replication did not have a strong effect on the microbiome composition. \\ Presence of CMV DNA (but not EBV) was associated with up-regulated expression of selected \\ cytokines in the gut of HIV-uninfected individuals. These results highlight a possible modulatory \\ effect of CMV on the gut immune homeostasis.
}

\section{BOOSTING NORMAL HOMEOSTASIS TO EXPEDITE ELIMINATION OF HIV-INFECTED MEMORY T CELLS IN PATIENTS ON ART: A POTENTIAL STRATEGY FOR CURE?}

\begin{abstract}
Author
Zvi Grossman

Lead Author's Institution

NIAID, National Institutes of Health, Bethesda, Maryland

Summary

Under effective ART, HIV DNA-containing CD4 T cells would be progressively washed out along with their uninfected counterparts if an influx of new memory cells can generated, in a controlled manner, by activation of naïve cells, while existing memory cells that are simultaneously activated preferentially differentiate and die. This strategy may be tuned for maximum efficiency and minimal side effects and lead to a functional cure. In a proof-of-principle study, the feasibility of inducing substantial washout of existing CD4+ memory is being tested in the mouse model.
\end{abstract}

\section{MICROBIAL INTERACTIONS AND PHARMACOMICROBIOMICS IN HIV TRANSMISSION}

\section{Authors}

Nichole Klatt, Ryan Cheu, Alex Zevin, Adam Burgener 


\section{Lead Author}

Nichole Klatt

Lead Author's Institution

University of Washington, Seattle, Washington

\section{Summary}

Microbiota can affect HIV transmission by many mechanisms, including by altering inflammation, barrier function, and most recently, we have shown that microbiota can directly metabolize antiretroviral drugs. Here we presented data on how dysbiotic vaginal bacteria can affect the pharmacologically active form of tenofovir.

\section{VIRAL DYNAMICS: DÉJÀ VU ALL OVER AGAIN}

\section{Author}

Jeffrey Lifson

\section{Lead Author's Institution}

AIDS and Cancer Virus Program, Leidos Biomedical Research, Inc., Frederick, Maryland; Frederick National Laboratory for Cancer Research, Frederick, Maryland

\section{Summary}

Certain combinations of the timing of cART initiation and its duration appear to be capable of facilitating off-cART host control of viral replication in nonhuman primate models. The potential mechanisms underlying this enhanced control, and its potential clinical relevance were discussed.

\section{FROM JUNK SCIENCE TO SCIENCE ON JUNK: EXTRACELLULAR VESICLES AND HIV}

\section{Authors}

Anush Arakelyan, Wendy Fitzgerald, Sonia Zicari, Christophe Vanpouille, Leonid Margolis

\section{Lead Author}

Leonid Margolis

\section{Lead Author's Institution}

Eunice Kennedy Shriver National Institute of Child Health and Human Development, National Institutes of Health, Bethesda, Maryland

\section{Summary}

Extracellular vesicles are produced by virus-infected cells and are part of viral preparations. HIV1 infected cells release extracellular vesicles that carry Env (gp120) and facilitate HIV-1 infection 


\section{TARGETING TIGIT AS A PATHWAY TO THE ERADICATION OF HIV}

\section{Authors}

Glen M. Chew, Ivo Sah Bandar, Michael Corley, Steven Deeks, Jonah Sacha, Cecilia M. Shikuma, Lishomwa C. Ndhlovu

\section{Lead Author}

Lishomwa C. Ndhlovu

Lead Author's Institution

John A. Burns School of Medicine, University of Hawaii, Honolulu, Hawaii

\section{Summary}

Strategies to eliminate the HIV viral reservoir to date have not succeeded. The TIGIT immune regulatory pathway when blocked may restore anti-HIV immune function. Here we discuss combinatorial adjunctive immunomodulatory approaches targeting the TIGIT pathway capable of depleting latently HIV infected T cells in blood and tissue sanctuaries.

\section{IMMUNE RECONSTITUTION AND SKEWED RESPONSES AFTER ART INITIATION IN HIV INFECTED UGANDANS}

\section{Authors}

Krystelle Nganou-Makamdop, Aarthi Talla, Sam Darko, Jeffrey Chipman, Greg Beilman, Torfi Hoskuldsson, Thomas Schmidt, Cissy Kityo, Rafick Sekaly, Timothy Schacker, Daniel Douek

\section{Lead Author}

Krystelle Nganou- Makamdop

\section{Lead Author's Institution}

Vaccine Research Center NIAID NIH, Human Immunology Section, Bethesda, Maryland

\section{Summary}

Longitudinal evaluation of plasma markers, transcriptome of sorted PBMC and circulating microbiome after initiation of ART in HIV infected Uganda was presented.

\section{HYPERCOAGULATION AS A SOURCE OF IMMUNE ACTIVATION AND INFLAMMATION DURING SIV INFECTION}

\section{Authors}

Ivona Pandrea, Rusell Tracy, Cristian Apetrei, Irini Sereti

\section{Lead Author}

Ivona Pandrea 


\title{
Lead Author's Institution
}

University of Pittsburgh, Pittsburgh, Pennsylvania; National Institutes of Health, Bethesda, Maryland

\section{Summary}

We presented data that support a bidirectional relationship between coagulation and immune activation/inflammation. We also confirmed that (Tissue Factor) TF is situated at the crossroads of the coagulation and inflammation vicious circle and showed results that suggest that TF may be an attractive therapeutic target to prevent chronic inflammation and disease progression.

\section{TELMISARTAN DOES NOT IMPROVE LYMPH NODE OR FAT FIBROSIS IN TREATED HIV INFECTION MORE THAN ART ALONE}

\section{Authors}

Netanya S Utay, Doug Kitch, Carl Fichtenbaum, Michael M Lederman, Jacob D Estes, Clara Magyar, Karen L Klingman, Judy S Currier and Jordan E Lake for the A5317 team

\author{
Lead Author \\ Netanya Utay \\ Lead Author's Institution \\ University of Texas Medical Branch, Galveston, Texas
}

\section{Summary}

In HIV-1-infected adults on suppressive ART, angiotensin receptor blockade and PPAR- $\gamma$ agonism with telmisartan for 48 weeks did not improve lymph node or adipose tissue fibrosis more than continued ART alone. Notably, continued ART decreased both lymph node and adipose tissue fibrosis over 48 weeks.

\section{LOSS OF MITOCHONDRIAL ACTIVITY IN CYCLING EFFECTOR REGULATORY T CELLS IS ASSOCIATED WITH PERTURBATION IN T CELL HOMEOSTASIS IN TREATED HIV-1 INFECTION}

\section{Authors}

Souheil-Antoine Younes, Aarthi Talla, Susan Pereira Ribeiro, Evgeniya V. Saidakova, Larisa B. Korolevskaya, Konstantin V. Shmagel, Carey L. Shive, Robert Balderas, Leonid Margolis, Daniel C. Douek, Donald. D. Anthony, Pushpa Pandiyan, Scott F. Sieg, Michael M. Lederman, and Rafick-Pierre Sekaly

\section{Lead Author}

Souheil-Antoine Younes

Lead Author's Institution

Case Western Reserve University, Cleveland, Ohio 


\section{Summary}

In this study, we show that perturbations in the homeostasis of CD4 T cells in HIV infected patients who fail to restore CD4 T cells on ART is associated with poor maintenance of regulatory $\mathrm{T}$ cells that is linked to impaired mitochondrial function.

\section{References}

1. Check E. Bad Boys question received wisdom on HIV. Nature. 2006;443(7110):380-1. PubMed PMID: 17006482. doi: 10.1038/443380b 